\title{
Microbiological changes after periodontal therapy in diabetic patients with inadequate metabolic control
}

\section{Carina Maciel SILVA- BOGHOSSIAN(a)}

Silvana Regina Perez ORRICO(b) Daniela GONÇALVES(b)

Fernanda Oliveira Bello CORREA ${ }^{(b)}$ Ana Paula Vieira COLOMBO(a)

(a) Department of Medical Microbiology, Institute of Microbiology Paulo de Goes, Universidade Federal do Rio de Janeiro UFRJ, Rio de Janeiro, RJ, Brazil.

(b)Department of Diagnosis and Surgery, Faculdade de Odontologia de Araraquara, Univ Estadual Paulista - UNESP,

Araraquara, SP, Brazil.

Declaration of Interests: The authors certify that they have no commercial or associative interest that represents a conflict of interest in connection with the manuscript.

Corresponding Author:

Carina Maciel Silva-Boghossian

E-mail: carinabogho@hotmail.com

DOI: 10.1590/1807-3107BOR-2014.vol28.0007 Epub: XXX XX, 2014

Submitted: Dec 11, 2012

Accepted for publication: Dec 11, 2013

Last revision: Jan 28, 2014

\begin{abstract}
The present study investigated the effect of non-surgical periodontal treatment (SRP) on the composition of the subgingival microbiota of chronic periodontitis (CP) in individuals with type 2 diabetes (DM2) with inadequate metabolic control and in systemically healthy (SH) individuals. Forty individuals (20 DM2 and $20 \mathrm{SH}$ ) with CP underwent full-mouth periodontal examination. Subgingival plaque was sampled from 4 deep sites of each individual and tested for mean prevalence and counts of 45 bacterial taxa by the checkerboard method. Clinical and microbiological assessments were performed before and 3 months after SRP. At baseline, those in the DM2 group presented a significantly higher percentage of sites with visible plaque and bleeding on probing compared with those in the SH group $(p<0.01)$. Those in the DM2 group presented significantly higher levels of $C$. rectus and $P$. gingivalis, and lower prevalence of $P$. micra and $S$. anginosus, compared with those in the SH group $(p \leq 0.001)$. At the 3-month visit, both groups showed a significant improvement in all clinical parameters $(p<0.01)$. Those in the DM2 group showed significantly higher prevalence and/ or levels of A. gerencseriae, A. naeslundii I, A. oris, A. odontolyticus, C. sputigena, F. periodonticum, and G. morbillorum compared with those in the $\mathrm{SH}$ group $(p \leq 0.001)$. However, those in the DM2 group showed a significant reduction in the levels of $P$. intermedia, $P$. gingivalis, T. forsythia, and T. denticola $(p \leq 0.001)$ over time. Those in the SRP group showed improved periodontal status and reduced levels of putative periodontal pathogens at 3 months' evaluation compared with those in the DM2 group with inadequate metabolic control.
\end{abstract}

Keywords: Diabetes Mellitus; Periodontal Debridement; Bacteria.

\section{Introduction}

Chronic hyperglycemia in diabetes mellitus (DM) is associated with an increased risk of development of systemic complications over the years, including microangiopathy, neuropathy, nephropathy, microvascular disease, and delayed wound-healing. ${ }^{1}$ Moreover, patients with DM have worse periodontal conditions compared with their non-diabetic counterparts. ${ }^{2,3}$ Persons with poorly controlled DM also present more severe periodontal disease than do those with well-controlled DM.,5 Several mechanisms have been proposed to explain the periodontal etiopathogenesis in patients with DM. ${ }^{3,6}$ Significant differences may be present in 
the periodontal microbiota between diabetic and non-diabetic individuals with chronic periodontitis (CP)., ${ }^{3,7,9}$ However, there are conflicting findings which have shown that poorly controlled insulindependent individuals with DM may have elevated levels of microbiota, ${ }^{10}$ lower prevalence of pathogenic species, ${ }^{8}$ or even a microbiota similar to that of nonDM individuals. ${ }^{2,11}$ Differences among studies may be explained by the metabolic control used. ${ }^{12,13}$ Scaling and root planing (SRP) therapy, in combination (or not) with antimicrobial agents, might be effective in reducing periodontal pathogens in persons with $\mathrm{DM}_{1}^{14,15}$ although others have reported that SRP alone may not be capable of eliminating or reducing Tannerella forsythia and Porphyromonas gingivalis in those individuals. 4,8,16,17,18 In general, a reduced level of those pathogens is crucial for a good response to therapy. ${ }^{19,20}$ Analysis of data in the literature shows that it is unclear whether the subgingival microbiota in persons with DM is significantly affected by SRP. Therefore, the aim of the present study was to investigate the effect of SRP on the composition of the subgingival microbiota in individuals with $\mathrm{CP}$ and DM type 2 with inadequate metabolic control (DM2), and in systemically healthy patients (SH).

\section{Methodology Study Population}

The study population has been described in a previous report. ${ }^{21}$ The participants were recruited at the Faculdade de Odontologia de Araraquara of the Universidade Estadual Paulista (FOAR/Unesp), Brazil. Informed consent was obtained from all individuals. Twenty participants ( 12 females; mean age, $45.80 \pm 6.01$ years) had DM2, ${ }^{1}$ inadequate glycemic control ${ }^{22}$ (glycated hemoglobin A1c-HbA1c $\geq 7 \%$ ), and mean DM duration of $9.85 \pm 7.10$ years; and $20 \mathrm{SH}$ participants (10 females; mean age, $43.65 \pm 6.01$ years) had no $\mathrm{DM}$, as confirmed by a fasting plasma glucose test. ${ }^{1}$ The patients did not receive any instruction on glycemia control, to avoid a bias due to interference in the effect of the periodontal treatment. Also, there was no intervention by doctors regarding medication. The sample size was calculated based on a pilot study. It was estimated that, with a minimum of 20 participants per group, significant differences in the clinical data would be detected between the study periods with $80 \%$ statistical power and a $95 \%$ confidence interval (unpublished data). The sample size calculation for microbiological analysis was based on a large microbiological database of over 400 individuals in our population evaluated over 8 years. That sample size calculation was performed considering a difference of $1 \times 10^{4} \pm 0.9 \times 10^{4}$ cells in the reduction of mean counts of the main outcome variable, "red complex" (Porphyromonas gingivalis, Treponema denticola, and Tannerella forsythia), ${ }^{23}$ between groups after therapy. ${ }^{19,24}$ A number of 14 individuals was estimated for each group with an error of $5 \%$ and a power of $80 \%$. The study protocol was approved by the Ethics in Human Research Committee of the FOAR-UNESP (no. 85/04). All participants had a diagnosis of chronic periodontitis, ${ }^{25}$ at least 15 teeth (except third molars), and moderate to severe $\mathrm{CP}$-i.e., at least 4 teeth with one or more sites with probing depth $(\mathrm{PD}) \geq 5 \mathrm{~mm}$, clinical attachment level (CAL) $\geq 4 \mathrm{~mm}$, and bleeding on probing (BOP). Exclusion criteria included pregnancy, use of contraceptives or other female hormones, smoking or cessation of smoking for $\leq 5$ years, use of local or systemic antimicrobial agents 6 months and antiinflammatory drugs 3 months prior to the study, and periodontal treatment in the preceding year. Participants with DM2 were under the supervision of an endocrinologist, with no alteration in DM treatment in the year prior to the study. Glycemic control was evaluated by the \%HbA1c with high-performance liquid chromatography (DiaSTAT Hemoglobin A1c Analyzer System, BioRad Lab., Hercules, USA) at baseline, immediately before and 3 months after SRP.

\section{Clinical assessment and therapy}

Periodontal measurements were performed by one calibrated examiner (kappa $=0.90$; D.G.), who was blinded for group assignment, using a manual periodontal probe (North Carolina Probe, Hu-Friedy, Chicago, USA) at 6 sites per tooth in all teeth, except third molars, at baseline and 3 months after SRP. Measurements included PD and CAL, BOP, suppuration on probing (SUP), and visible plaque (VPI) and gingival marginal bleeding (GBI) indices. The SRP was performed by a single experienced periodon- 
tist (F.O.B.C.) who was not aware of the group distribution, as follows: oral hygiene instructions and 4 weekly sessions of SRP (1 hour each) under local anesthesia, and professional plaque control twice a month for 3 months. All participants returned on the scheduled days for professional plaque control.

\section{Microbiological assessment}

The prevalence and levels of 45 bacterial taxa were determined by the checkerboard DNA-DNA hybridization method. ${ }^{23,26}$ After removal of supragingival biofilm, subgingival biofilm samples were taken from 4 deep sites (characterized as $P D \geq 5 \mathrm{~mm}$, $\mathrm{CAL} \geq 4 \mathrm{~mm}$, and $\mathrm{BOP}$ ) in different non-adjacent teeth at baseline and at the 3-month visit after SRP, with sterilized Gracey curettes (Trinity Periodontia, São Paulo, Brazil) and placed in individual tubes. Bacterial cells were subjected to lysis by the addition of $0.5 \mathrm{M} \mathrm{NaOH}$ and boiling, and denatured DNA was fixed on a nylon membrane (GE Healthcare Life Science, São Paulo, Brazil) by means of the Minislot 30 device (Immunetics, Cambridge, USA). The membrane was placed in a Miniblotter 45 (Immunetics) with the lanes of DNA at $90^{\circ}$ to the lanes of the device, and hybridized against digoxigenin-labeled (Roche Applied Science, São Paulo, Brazil) wholegenomic DNA probes for the selected species. After hybridization, the membranes were washed at high stringency, bound probes were detected with phosphatase-conjugated antibody to digoxigenin (Roche Applied Science), and fluorescence was captured by an imaging system (Storm ${ }^{\mathrm{TM}}$ 860, GE Healthcare Life Science, São Paulo, Brazil). Signals were evaluated visually by comparison with the standards at $10^{5}$ and $10^{6}$ bacterial cells for the test species on the same membrane. They were recorded as: 0 , not detected; $1,<10^{5}$ cells; 2 , approximately $10^{5} ; 3,10^{5}-10^{6}$ cells; 4 , approximately $10^{6}$; and $5,>10^{6}$ cells.

\section{Statistical analysis}

The participant was the unit of analysis in a statistical program (IBM SPSS Statistics Version 19, IBM, Armonk, USA). Clinical parameters and the microbiological data were calculated for each participant and then across the group. Microbial data were expressed as mean \% of colonized sites (prev- alence), and mean counts (mean of bacterial cells) were $\log ^{10}$-transformed for each species. ${ }^{23}$ The mean counts were determined by transforming the scores 0 to 5 in bacterial counts. Clinically significant differences between groups were determined by the Mann-Whitney test. Significant differences in microbiological parameters between groups at baseline were determined by the Mann-Whitney test. Differences in microbiological changes in diseased sites within and between groups over time were evaluated by General Linear Models (GLM) for repeated measures. Any difference between groups detected in the period of reference was controlled in the multivariate model by GLM evaluation. Significant differences within groups over time were calculated by the Wilcoxon signed-rank test. For microbial analysis, adjustments were made for multiple comparisons as described by Socransky et al. ${ }^{27}$ In brief, an overall $p$ of $0.05=1-(1-k)^{45}$ was computed, where $\mathrm{k}$ was the desired individual $p$ value. Thus, a $p$ value $\leq 0.001$ was considered to be statistically significant at $p<0.05$.

\section{Results}

There were no significant differences regarding gender and age between groups (data not shown). The glycemic control in those with DM2 showed no significant reduction in $\mathrm{HbA1c}$ mean values from baseline $(9.43 \pm 1.80)$ to the 3 -month visit $(9.03 \pm 1.94$; $p=0.79$, Wilcoxon test; data not shown). At baseline, those with DM2 presented significantly higher mean percentages of VPI and BOP compared with those in the SH group ( $p<0.001$, Mann-Whitney test; Table 1). At the 3-month visit, both groups showed a significant improvement in all clinical parameters $(p<0.01$, GLM test). Those in the SH group showed a significant reduction in VPI compared with those with $\mathrm{DM} 2$, while the latter showed a greater reduction in mean CAL than the former over time $(p<0.05$, GLM test). For further information regarding clinical data, please refer to Gonçalves et al. ${ }^{21}$

At baseline, those in the $\mathrm{SH}$ group harbored a greater number of species with high prevalence (> 50\% of the sampled sites) than those with DM2, including various species of streptococci, Fusobacterium nucleatum, Prevotella intermedia, members of the red complex (P. gingivalis, Treponema denticola, $T$. 
Table 1. Mean $( \pm S D$ ) of the periodontal clinical parameters for the control $(S H)$ and diabetes (DM2) groups at baseline and 3 months after SRP.

\begin{tabular}{lcccc}
\hline \multirow{2}{*}{ Clinical Parameters } & \multicolumn{2}{c}{$\mathrm{SH}(\mathrm{N}=20)$} & \multicolumn{2}{c}{$\mathrm{DM} 2(\mathrm{~N}=20)$} \\
\cline { 2 - 5 } & Baseline & 3 months & Baseline & $3 \mathrm{months}$ \\
\hline VPI (\%) $)^{* \dagger}$ & $65.3 \pm 14.9$ & $8.1 \pm 12.8^{\dagger}$ & $85.6 \pm 11.3$ & $17.9 \pm 14.2^{\dagger}$ \\
GBI (\%) & $38.4 \pm 16.5$ & $2.9 \pm 2.3^{\dagger}$ & $47.3 \pm 23.2$ & $10.8 \pm 10.4^{\dagger}$ \\
BOP (\%)* & $76.1 \pm 16.1$ & $15.8 \pm 10.7^{\dagger}$ & $90.9 \pm 9.9$ & $28.2 \pm 15.9^{\dagger}$ \\
SUP (\%) & $7.5 \pm 7.1$ & $0.2 \pm 0.5^{\dagger}$ & $11.6 \pm 9.5$ & $0.9 \pm 1.7^{\dagger}$ \\
PD (mm) & $3.6 \pm 0.6$ & $2.4 \pm 0.3^{\dagger}$ & $4.2 \pm 0.9$ & $2.7^{\dagger} \pm 0.4^{\dagger}$ \\
CAL (mm) & $4.3 \pm 1.0$ & $3.9 \pm 0.8^{\dagger}$ & $4.9 \pm 1.5$ & $4.3 \pm 1.3^{\dagger}$ \\
PD $\geq 5 \mathrm{~mm}(\%)$ & $27.9 \pm 11.2$ & $4.2 \pm 4.2^{\dagger}$ & $39.1 \pm 24.2$ & $7.7 \pm 7.3^{\dagger}$ \\
$\mathrm{CAL} \geq 5 \mathrm{~mm}(\%)$ & $42.3 \pm 18.5$ & $36.4 \pm 21.3^{\dagger}$ & $51.1 \pm 24.7$ & $43.6 \pm 24.6^{\dagger}$ \\
\hline
\end{tabular}

${ }^{*} p<0.05$, between SH and DM2 at baseline (Mann-Whitney test;) ${ }^{\dagger} p<0.001$, between baseline and 3 months after SRP (GLM); ${ }^{\ddagger} p<0.05$, between groups over time (GLM). VPI, visible plaque; GBI, gingival marginal bleeding; SUP, suppuration on probing; $B O P$, bleeding on probing; PD, probing depth; CAL, clinical attachment level.

forsythia), and Neisseria mucosa (Figure 1). However, only Parvimonas micra and Streptococcus anginosus presented significantly higher prevalence in those in the $\mathrm{SH}$ group compared with those in the DM2 group ( $p \leq 0.001$, Mann-Whitney test). At the 3-month visit, those with DM2 showed significantly higher prevalence of Actinomyces naeslundii I, Actinomyces oris, Actinomyces odontolyticus, and Gemella morbillorum than those in the SH group ( $p \leq 0.001)$. Changes over time within groups showed that those in the $\mathrm{SH}$ group had a significant decrease in species of the orange (F. nucleatum polymorphum, F. nucleatum nucleatum, P. intermedia, and Prevotella nigrescens) and red (P. gingivalis, T. forsythia, T. denticola) complexes, Aggregatibacter actinomycetemcomitans $a$, and N. mucosa (adjusted $p \leq 0.001$, Wilcoxon test), whereas those with DM2 had a significant decrease only in the members of the red complex and G. morbillorum ( $p \leq$ 0.001). In general, both groups showed a reduction in bacterial load (Figure 2). At baseline, those with DM2 presented significantly higher levels of Campylobacter rectus and $P$. gingivalis and lower levels of $P$. micra and S. anginosus compared with SH individuals (adjusted $p \leq 0.001$, Mann-Whitney test). After therapy, those with DM2 harbored significantly higher levels of Actinomyces gerencseriae, A. odontolyticus, A. naeslundii I, A. oris, Capnocytophaga sputigena, Fusobacterium periodonticum, and G. morbillorum (adjusted $p \leq$ 0.001 ) than SH individuals. Significant reductions in mean levels of several periodontal pathogens were seen in SH individuals over time (adjusted $p \leq 0.001$,
Wilcoxon test). Conversely, only P. intermedia, P. gingivalis, T. forsythia, and T. denticola showed a significant decrease in mean levels in those with DM2 after therapy (adjusted $p \leq 0.001$ ). In addition, there was a significant increase from baseline to the 3-month visit in the levels of Staphylococcus aureus in those with DM2 (adjusted $p \leq 0.001$ ).

\section{Discussion}

Patients with DM and poor glycemic control present more severe gingival inflammation and periodontal destruction compared with individuals without DM and/or those with well-controlled DM.,11,28 In the current study, those with DM2 and inadequate metabolic control presented significantly higher extensions of VPI and BOP than did SH individuals at baseline. Although those with DM2 presented greater values for other inflammation parameters and periodontal destruction, no significant differences between groups were observed. SRP resulted in significant improvements in all clinical parameters in both groups, corroborating results from previous studies. ${ }^{6,8,14,28}$ Significant differences between groups were seen for percentages of VPI and mean CAL. Despite a rigorous periodontal maintenance program and a considerable clinical improvement after treatment, those with DM2 still presented higher percentages of sites with VPI than did SH individuals.

Periodontal disease may affect those with DM directly through chronic inflammatory alterations. ${ }^{29}$ The presence of inflammation plays an important 
$\mathrm{SH}(\mathrm{n}=20)$

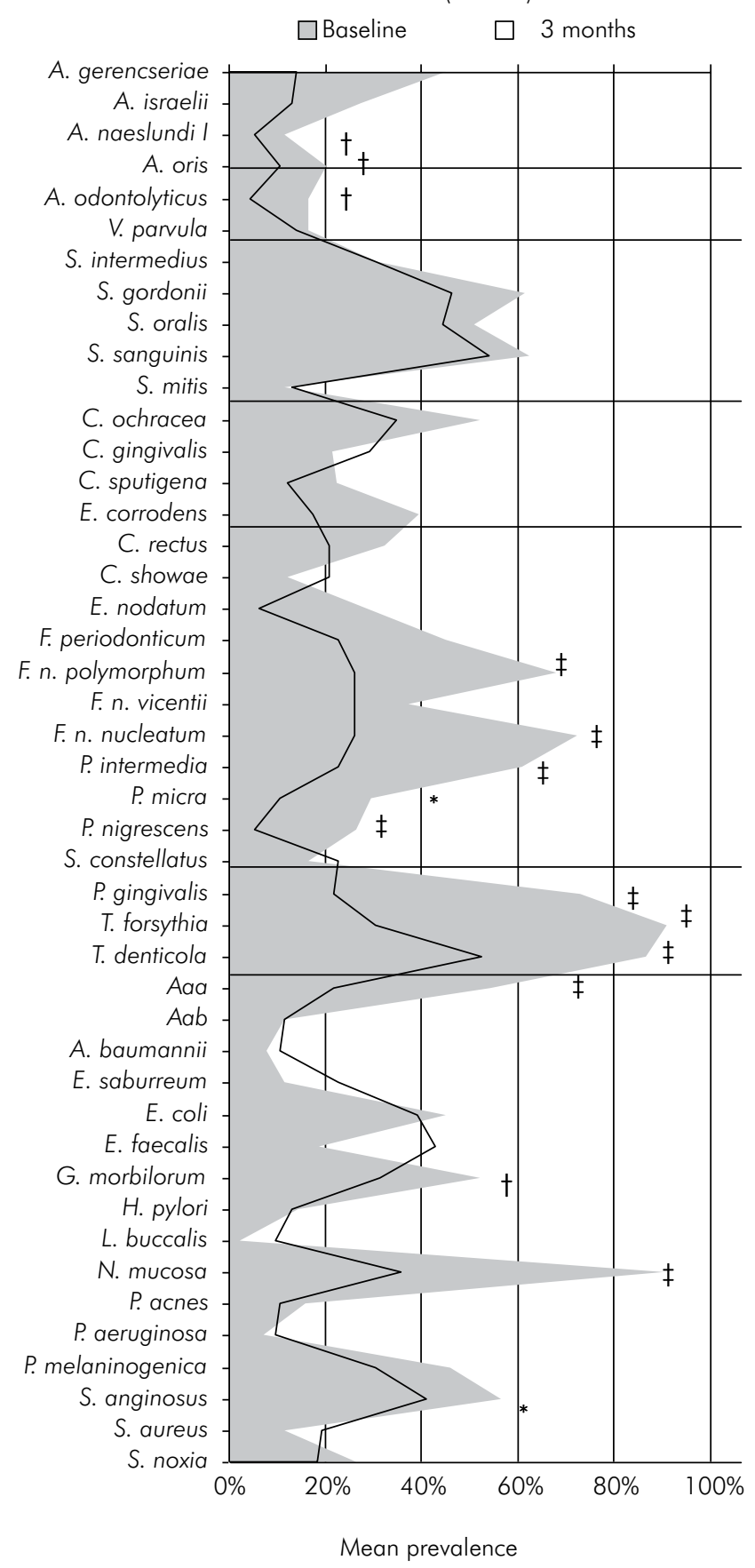

$\mathrm{DM} 2(\mathrm{n}=20)$

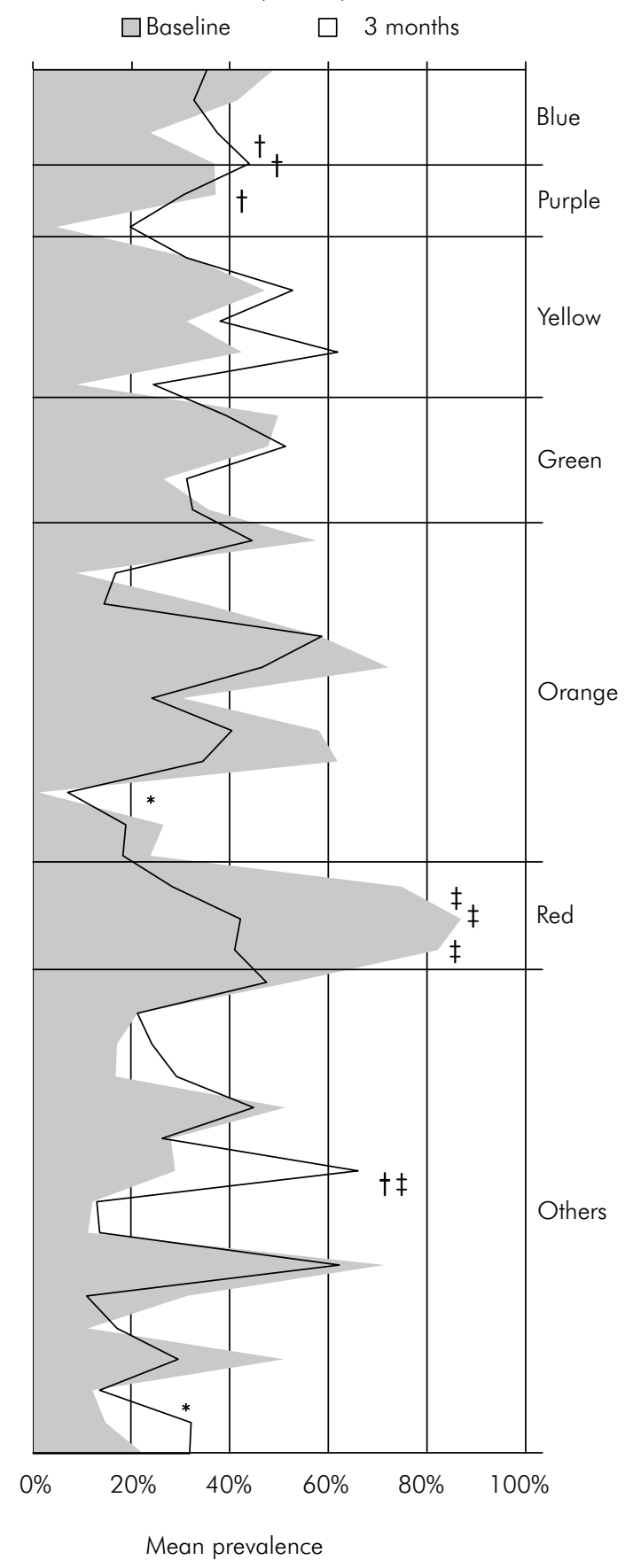

*Significant differences between groups at baseline (adjusted $p \leq 0.001$, Mann-Whitney test). tSignificant differences between groups at 3 months post-therapy (adjusted $p \leq 0.001$, Mann-Whitney test). FSignificant differences within the groups over time (adjusted $p \leq 0.001$, Wilcoxon test).

Figure 1. Mean prevalence of subgingival species evaluated in biofilm samples from individuals with type 2 diabetes (DM2) and systemically healthy $(\mathrm{SH})$ individuals with chronic periodontitis, at baseline and 3 months after SRP. The species were ordered according to the bacterial complexes. 25 Aa: Aggregatibacter actinomycetemcomitans.

role in insulin sensitivity and glucose dynamics. ${ }^{30}$ Clinical studies have demonstrated that improvement in metabolic control correlates with improve- ment in periodontal health. ${ }^{15,31,32}$ However, in the current study, while the improvement in the periodontal condition observed in those with DM had some 

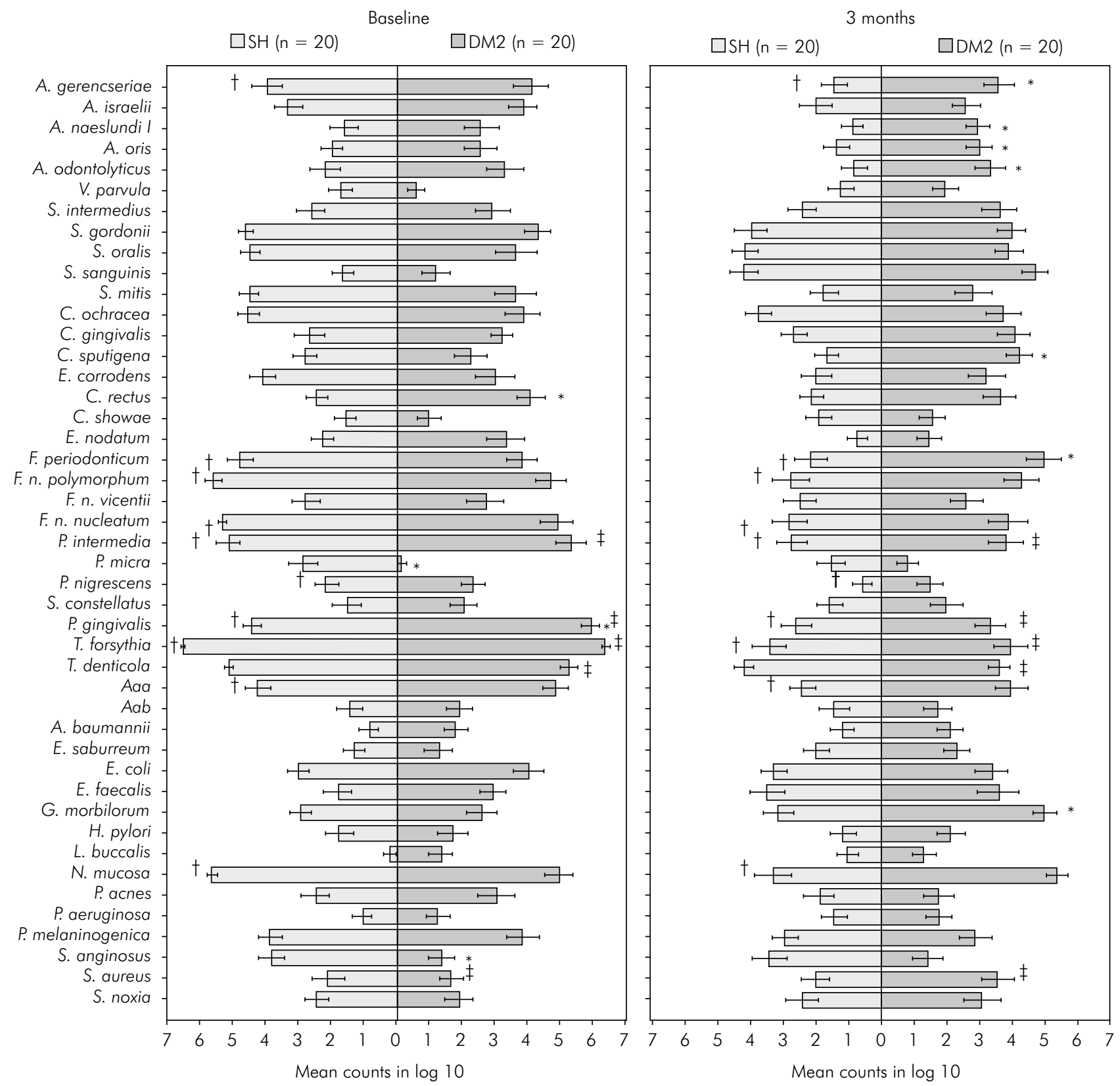

*Significant differences between groups at baseline or at 3 months after SRP (adjusted $p \leq 0.001$, Mann-Whitney test). tSignificant differences within the SH group over time (adjusted $p \leq 0.001$, Wilcoxon test). .Significant differences within the DM2 group over time (adjusted $p \leq 0.001$, Wilcoxon test).

Figure 2. Mean counts (in log $10 \pm$ SEM) of subgingival species evaluated in biofilm samples from individuals with type 2 diabetes (DM2) and systemically healthy (SH) individuals with chronic periodontitis at baseline (left panel) and 3 months (right panel) after SRP. Aa: Aggregatibacter actinomycetemcomitans.

impact on glycemic control, it did not reach statistical significance. Moreover, glycemic control is influenced by other variables, such as diet, weight control, physical exercises, and use of medication to control glycemic level. So that bias would be avoided, none of those variables underwent any alteration during the study, so the obtained effect might simply be a result of the periodontal treatment.

Analysis of microbiological data at baseline showed that both groups presented high mean counts and prevalence of periodontal pathogens, such as members of the red and orange complexes. 
However, those with DM2 presented significantly higher bacterial levels of $C$. rectus and $P$. gingivalis compared with SH individuals. Mandell et al.$^{10}$ also observed increased levels and prevalence of $C$. rec$t u s$, as well as other periodontal pathogens, at diseased sites in those with poorly controlled insulindependent DM. Other species, such as $P$. gingivalis, $A$. actinomycetemcomitans, Campylobacter spp., Capnocytophaga spp., and Eubacterium nodatum, can be detected at high frequency in those with DM.,7,9 Conversely, Cruz et al. ${ }^{8}$ observed a low frequency of sites with T. forsythia in those with DM. In accordance with other studies, ${ }^{2,11}$ Field et al. ${ }^{33}$ demonstrated that the amounts of A. actinomycetemcomitans, F. nucleatum, and $P$. gingivalis did not differ between individuals with periodontitis, with and without DM. Hintao et al. ${ }^{11}$ did not detect any significant difference in 17 subgingival species using the checkerboard method in participants with and without DM.

Analysis of baseline data showed that those with DM2 had a 'worse' microbiological profile when compared with those in the SH group; nevertheless, this was not a key factor preventing a good clinical response to SRP. Overall, the microbiological findings regarding changes in bacterial prevalence and levels over time demonstrated that a greater number of species were reduced in $\mathrm{SH}$ individuals than in those with DM2, although many of those species are considered beneficial. ${ }^{18,20}$ Significant differences between groups were observed only for Actinomyces spp., C. sputigena, F. periodonticum, and G. morbillorum. Even though more species diminished in $\mathrm{SH}$ individuals after therapy, pathogenic species had a significant decrease in prevalence and/or levels, while host-compatible species such as Actinomyces spp. and G. morbillorum were increased in those with DM2. That is in agreement with results from studies showing that SRP can lead to a shift from pathogenic to beneficial species in the subgingival environment. ${ }^{19,26}$ The levels of the species $S$. aureus, which can be associated with periodontitis, ${ }^{34}$ increased significantly in those with DM2 after SRP. Again, longitudinal analysis showed that individuals with DM2 presented a different microbiological profile compared with $\mathrm{SH}$ individuals after therapy. As mentioned previously, differences in microbiological profile in those with DM2 can be due to the impaired inflammatory response, which can lead to a favorable environment for proteolytic species. ${ }^{17}$ Conversely, a differentiated prognosis should always be considered, not only because of the inflammatory characteristics of DM2, but also due to their microbiological features. As a result, close attention should be given to individuals with DM2 during the maintenance phase. Moreover, species considered to be host-compatible, such as Actinomyces spp. and Streptococcus spp., showed no significant variations from baseline to the 3-month visit. The majority of Streptococcus spp. and a few species of the genus Actinomyces presented a modest increase in counts and prevalence after treatment in both groups. These results are in agreement with those from other studies demonstrating that mechanical treatment can result in a shift from pathogenic to beneficial species in the subgingival environment. ${ }^{19,26}$ The results found in the group with DM2 regarding this matter are important, because they show that individuals with DM2 are also able to present an increase in the levels of those species that will help in the maintenance of a balanced subgingival environment. Mechanical periodontal therapy alone may not be capable of eliminating $T$. forsythia and $P$. gingivalis in individuals with DM. ${ }^{35}$ Other studies reported a reduction in the prevalence of these pathogens when SRP was used in combination with antimicrobials. ${ }^{14,15}$ In the present study, those species were not eliminated but had significantly reduced levels, which enabled a good clinical response to be obtained, at least at 3 months after treatment in both groups. In contrast, other studies ${ }^{8,28}$ observed a modest and non-significant reduction in the levels of these species after full-mouth subgingival debridement in individuals with DM. However, these authors used only one or two sessions in contrast to four sessions of SRP and a rigorous periodontal maintenance program as in the present study.

\section{Conclusion}

SRP associated with a rigorous maintenance program improved the periodontal status and reduced the levels of putative periodontal pathogens at 3 months' evaluation in individuals with DM2 and inadequate metabolic control compared with $\mathrm{SH}$ individuals. 


\section{Acknowledgements}

The authors are thankful to Prof. Dr. José Govone. This study was supported in part by Coordenação de Aperfeiçoamento de Pessoal de Nível Superior (Capes
- Brasília, Brazil), Fundação de Amparo à Pesquisa do Estado de São Paulo (FAPESP - Grant no. 04/08142-0; São Paulo, Brazil), and Foundation for the Development of UNESP (Grant no. 00006/05-DFP; São Paulo).

\section{References}

1. American Diabetes Association. Diagnosis and classification of diabetes mellitus. Diabetes Care. 2008 Jan;31(Suppl 1):55-60.

2. Campus G, Salem A, Uzzau S, Baldoni E, Tonolo G. Diabetes and periodontal disease: a case-control study. J Periodontol. 2005 Mar;76(3):418-25.

3. Lalla E, Kaplan S, Chang SM, Roth GA, Celenti R, Hinckley $\mathrm{K}$, et al. Periodontal infection profiles in type 1 diabetes. $\mathrm{J}$ Clin Periodontol. 2006 Dec;33(12):855-62.

4. Lalla E, Cheng B, Lal S, Kaplan S, Softness B, Greenberg E, et al. Diabetes-related parameters and periodontal conditions in children. J Periodontal Res. 2007 Aug;42(4):345-9.

5. Lim LP, Tay FB, Sum CF, Thai AC. Relationship between markers of metabolic control and inflammation on severity of periodontal disease in patients with diabetes mellitus. J Clin Periodontol. 2007 Feb;34(2):118-23.

6. Faria-Almeida R, Navarro A, Bascones A. Clinical and metabolic changes after conventional treatment of type 2 diabetic patients with chronic periodontitis. J Periodontol. 2006 Apr;77(4):591-8.

7. Ciantar M, Gilthorpe MS, Hurel SJ, Newman HN, Wilson M, Spratt DA. Capnocytophaga spp. in periodontitis patients manifesting diabetes mellitus. J Periodontol. 2005 Feb;76(2):194-203.

8. da Cruz GA, de Toledo S, Sallum EA, Sallum AW, Ambrosano GM, de Cassia Orlandi Sardi J, et al. Clinical and laboratory evaluations of non-surgical periodontal treatment in subjects with diabetes mellitus. J Periodontol. 2008 Jul; 79(7):1150-7.

9. Ebersole JL, Holt SC, Hansard R, Novak MJ. Microbiologic and immunologic characteristics of periodontal disease in Hispanic Americans with type 2 diabetes. J Periodontol. 2008 Apr;79(4):637-46.

10. Mandell RL, Dirienzo J, Kent R, Joshipura K, Haber J. Microbiology of healthy and diseased periodontal sites in poorly controlled insulin dependent diabetics. J Periodontol. 1992 Apr;63(4):274-9.

11. Hintao J, Teanpaisan R, Chonqsuvivatwong V, Ratarasan C, Dahlén G. The microbiological profiles of saliva, supragingival and subgingival plaque and dental caries in adults with and without type 2 diabetes mellitus. Oral Microbiol Immunol. 2007 Jun;22(3):175-81.

12. Seppaa B, Ainamo J. Dark field microscopy of the subgingival microflora in insulin-dependent diabetics. J Clin Periodontol. 1996 Feb;23(2):63-7.

13. Tervonen T, Oliver RC, Wolff LF, Bereuter J, Anderson L, Aeppli DM. Prevalence of periodontal pathogens with varying metabolic control of diabetes mellitus. J Clin Periodontol. 1994 Jul;21(6):375-9.
14. Christgau M, Palitzsch KD, Schmalz G, Kreiner U, Frenzel $S$. Healing response to non-surgical periodontal therapy in patients with diabetes mellitus: clinical, microbiological, and immunologic results. J Clin Periodontol. 1998 Feb;25(2):112-24.

15. Grossi SG, Skrepcinski FB, DeCaro T, Robertson DC, Ho AW, Dunford RG, et al. Treatment of periodontal disease in diabetics reduces glycated hemoglobin. J Periodontol. 1997 Aug;68(8):713-9.

16. Kolenbrander PE, Palmer RJ Jr, Rickard AH, Jakubovics NS, Chalmers NI, Diaz PI. Bacterial interactions and successions during plaque development. Periodontol 2000. 2006;42:47-79.

17. Socransky SS, Haffajee AD, Ximenez-Fyvie LA, Feres M, Mager D. Ecological considerations in the treatment of Actinobacillus actinomycetemcomitans and Porphyromonas gingivalis periodontal infections. Periodontol 2000. 1999;20:341-62.

18. Teles RP, Haffajee AD, Socransky SS. Microbiological goals of periodontal therapy. Periodontol 2000. 2006;42:180-218.

19. Colombo AP, Teles RP, Torres MC, Rosalém W, Mendes MC, Souto RM, et al. Effects of non-surgical mechanical therapy on the subgingival microbiota of Brazilians with untreated chronic periodontitis: 9-month results. J Periodontol. 2005 May;76(5):778-84.

20. Haffajee AD, Teles RP, Socransky SS. The effect of periodontal therapy on the composition of the subgingival microbiota. Periodontol 2000. 2006;42:219-58.

21. Gonçalves D, Correa FO, Khalil NM, de Faria Oliveira OM, Orrico SR. The effect of non-surgical periodontal therapy on peroxidase activity in diabetic patients: a case-control pilot study. J Clin Periodontol. 2008 Sep;35(9):799-806.

22. Intensive blood-glucose control with sulphonylureas or insulin compared with conventional treatment and risk of complications in patients with type 2 diabetes (UKPDS 33). UK Prospective Diabetes Study (UKPDS) Group. Lancet. 1998 Sep 12; 352(9131):837-53.

23. Socransky SS, Haffajee AD, Cugini MA, Smith C, Kent RL Jr. Microbial complexes in subgingival plaque. J Clin Periodontol. 1998 Feb;25(2):134-44.

24. Heller D, Varela VM, Silva-Senem MX, Torres MC, Feres-Filho EJ, Colombo AP. Impact of systemic antimicrobials combined with anti-infective mechanical debridement on the microbiota of generalized aggressive periodontitis: a 6-month RCT. J Clin Periodontol. 2011 Apr;38(4):355-64. 
25. Armitage GC. Development of a classification system for periodontal diseases and conditions. Ann Periodontol. 1999 Dec;4(1):1-6.

26. Haffajee AD, Cugini MA, Dibart S, Smith C, Kent RL Jr, Socransky SS. The effect of SRP on the clinical and microbiological parameters of periodontal diseases. J Clin Periodontol. 1997 May;24(5):324-34.

27. Socransky SS, Haffajee AD, Smith C, Dibart S. Relation of counts of microbial species to clinical status at the sampled site. J Clin Periodontol. 1991 Nov;18(10):766-75.

28. Lalla E, Kaplan S, Yang J, Roth GA, Papapanou PN, Green-

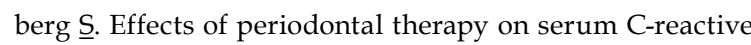
protein, sE-selectin, and tumor necrosis factor-alpha secretion by peripheral blood-derived macrophages in diabetes. A pilot study. J Periodontal Res. 2007 Jun;42(3):274-82.

29. Nassar H, Kantarci A, van Dyke TE. Diabetic periodontitis: a model for activated innate immunity and impaired resolution of inflammation. Periodontol 2000. 2007;43:233-44.
30. Mealey BL, Ocampo GL. Diabetes mellitus and periodontal disease. Periodontol 2000. 2007;44:127-53.

31. Kiran M, Arpak N, Unsal E, Erdoğan MF. The effect of improved periodontal health on metabolic control in type 2 diabetes mellitus. J Clin Periodontol. 2005 Mar;32(3):266-72.

32. Correa FO, Gonçalves D, Figueredo CM, Bastos AS, Gustafsson A, Orrico SR. Effect of periodontal treatment on metabolic control, systemic inflammation and cytokines in patients with type 2 diabetes. J Clin Periodontol. 2010 Jan;37(1):53-8.

33. Field CA, Gidley MD, Preshaw PM, Jakubovics N. Investigation and quantification of key periodontal pathogens in patients with type 2 diabetes. J Periodontal Res. 2012 Aug;47(4):470-8.

34. da Silva-Boghossian CM, do Souto RM, Luiz RR, Colombo AP. Association of red complex, A. actinomycetemcomitans and non-oral bacteria with periodontal diseases. Arch Oral Biol. 2011 Sep;56(9):899-906.

35. Smith GT, Greenbaum CJ, Johnson BD, Persson GR. Shortterm responses to periodontal therapy in insulin-dependent diabetic patients. J Periodontol. 1996 Aug;67(8):794-802. 